\title{
Um caso de opacidade fonológica na aquisição do PB: um problema ótimo ou uma questão exemplar?
}

\section{Giovana Ferreira Gonçalves}

Doutora em Linguística pela Pontifícia

Universidade Católica do Rio Grande do

Sul. Professora adjunta do Departamento de

Linguística da UFPel e professora colaboradora do Programa de Pós-Graduação em Letras da Universidade Federal de Santa Maria.

Resumo: Tendo em vista que a Teoria da Otimidade Standard avalia seus candidatos de forma paralela, banindo a serialidade, a opacidade fonológica é um dos fenômenos mais difíceis de ser abordado por esse enfoque teórico, justamente por ser oriunda da derivação. Embora muitas reformulações à TO tenham sido implementadas, nenhuma é capaz de formalizar todos os casos de opacidade existentes nas línguas do mundo. Nesta luz, advogamos que a opacidade deve ser tratada como uma questão da representação linguística adotada por certos modelos de formalização. Dessa forma, defendemos, como possibilidade, um modelo multirrepresentacional de representação linguística, a Teoria de Exemplares.

Palavras-chave: opacidade fonológica; aquisição; Teoria da Otimidade; Teoria de Exemplares

\author{
FELIPE FLORES KUPSKE
}

Aluno de mestrado do Programa de Pós-

Graduação em Letras da UFSM, bolsista CAPES.

\begin{abstract}
Since Optimality Theory evaluates its candidates through a parallel way it banishes seriality completely; therefore, phonological opacity is one of the language phenomena the model has a grate difficulty to approach as it is rooted in rule application. Even though a vast range of approaches to OT or new reformulations to its structure have been made, none of them can cope with all sorts of opacity. In that light, this paper advocates that the question of opacity should be treated as a linguistic representation adopted by some models of linguistic formalization; therefore, supporting the switch to exemplars models' way of representing language.
\end{abstract}

Keywords: phonological opacity; acquisition; Optimality Theory; Exemplars Model 



\section{Introdução}

Embora detalhes acerca de terminologias e princípios implementacionais da fonologia gerativa possam variar, McCarthy (1999, p. 01) afirma que a característica que define a fonologia baseada em regras é a "preeminence of the chronological metaphor", em que uma forma subjacente é transformada em uma sucessão de diferentes representações no percurso até a superfície, idéia que Chomsky e Halle (1968) denominam de derivação serial. Nas teorias operacionais ou transformacionais teorias baseadas em regras - generalizações podem ser (i) não-verdadeiras-na-superfície ou (ii) não-aparentes-nasuperfície. Opacidade, dessa forma, é o termo empregado na fonologia gerativa com referência ao fato de que uma determinada regra (generalização) a uma determinada forma não é percebida no resultado fonético final da derivação. (HYMAN, 1975).

Com o surgimento da Teoria da Otimidade, por Prince e Smolensky em 1993, é oferecida uma visão discutível e incompleta acerca da opacidade (COSTA, 2007). Neste modelo de análise teórica, que veio para reduzir os altos níveis de abstração dos modelos anteriores, as generalizações opacas são, nas palavras de Costa (Ibid, p. 14), "ainda mais instigantes, já que a arquitetura padrão dessa teoria não é capaz de derivar muitas delas", na verdade, nenhuma, caso venhamos a adotar o modelo standard de 1993, que não comporta derivação.

Enquanto para teorias derivacionais da fonologia o modo natural de lidar com a opacidade é o ordenamento de regras, a TO clássica, teoria inerentemente nãoderivacional, não possui tal opção, pois propõe que o mapeamento entre input/output é feito de uma forma direta, em paralelo e sem estágios intermediários. Sendo assim, a questão da opacidade representa um desafio para este modelo teórico, de forma que continua a se reformular, tendo em vista as críticas dirigidas a propostas como a Teoria da Simpatia (MCCARTHY, 1999), TOEstratal (KIPARSKY, 1998; 2000), Marcação Comparativa 
(MCCARTHY, 2003), TO-Serial (ITO E MESTER, 2003) e TO com Cadeias de Candidatos (MCCARTHY, 2007), entre outras. Contudo, segundo Costa (2007), "todas as propostas feitas até então são passíveis de críticas em alguns aspectos. Ademais, nenhuma delas é capaz de apreender todo o tipo de opacidade presente nas línguas do mundo". Dessa forma, de acordo com Tihonova (2009), a opacidade fonológica tem sido tomada como a questão mais importante da teoria fonológica contemporânea.

De acordo com Coetzee, Kager e Pater (2009), atualmente há uma tendência a se considerar metodologias experimentais como suporte às análises fonológicas. $\mathrm{O}$ uso do laboratório para a coleta e tratamento dos dados passa, pois, a ser fundamental para testar predições vindas da teoria fonológica e também para desenvolver a teoria fonológica, de forma que ela possa melhor lidar com os dados coletados nos experimentos. Tal fato conduz, inevitavelmente, a um repensar acerca da natureza das representações linguísticas. Neste sentido, também sinaliza Cristófaro-Silva (2008b).

Consideramos que este viés deve ser trazido para as discussões acerca da opacidade fonológica, já que essas podem ser resolvidas ao adotarmos um modelo de representação linguística compatível com achados das ciências do cérebro das últimas décadas. Um enfoque que tome a língua como inerentemente variável e gradual, como o modelo multirrepresentacional de exemplares, em detrimento de um viés categórico e cartesiano do componente linguístico. Assim, neste trabalho, trazemos uma análise via restrições, com base na Teoria da Otimidade Standard e na LPM-OT, de um caso de opacidade da aquisição da fonologia proposto por Matzenauer (2003) e, em contraponto, trazemos uma abordagem com base no modelo de exemplares, considerando os estudos de Cristófaro-Silva (2006, 2008b) e Cristófaro-Silva e Gomes (2007a, 2007b). 


\section{Opacidade fonológica: do serial ao paralelo}

A Teoria Gerativa Clássica (TGC) reconhece dois níveis de representação: a estrutura subjacente e a estrutura de superfície. As representações de superfície são derivadas pela aplicação linear de regras e, por essa razão, duas questões são centrais à teoria, já que a partir delas a gramática fonológica de uma língua é construída. (COSTA, 2007). Para poder limitar os tipos de estrutura e processos existentes nas diferentes línguas do mundo, "a TGC postulou regras específicas às línguas, responsáveis pelas transformações; restrições, que limitavam a aplicação dessas regras; e princípios, que só poderiam ser considerados universais caso não fossem violados em nenhum sistema linguístico existente”. (Ibid., p.17). Entretanto, segundo Kager (1999) em Costa (2007, p. 17),

When some universal principle is violated in the output of the grammar, then the characteristic way of explaining this was to set up an intermediate level of representation at which it is actually satisfied. Each grammatical principle thus holds at a specific level of description, and may be switched off at other levels. ${ }^{2}$

A violação desses princípios adotados pela teoria aumentava a gama de níveis intermediários. Como consequência, onerava-se consideravelmente a gramática fonológica com representações linguísticas extremamente abstratas. Além desse problema ligado à abstração, as discussões estiveram voltadas ao modo como as regras interagiam entre si. Assim, vários estudos começaram a discutir as relações de ordenamento que surgiam na interação entre diferentes regras. "Viu-se, então, que a disposição puramente linear do modelo não apreendia todas as interações existentes nas línguas naturais". (Ibid., p. 18).

Para os modelos baseados em regras, a opacidade surge naturalmente das interações entre fenômenos 
linguísticos. É o ordenamento estabelecido entre esses fenômenos que produz diferentes efeitos: o da transparência ou o da opacidade. Segundo McCarthy (1999), basicamente são dois os tipos de opacidade: a não-verdadeira-na-superfície, que é gerada por processos que aparentemente encontram contexto, mas não atuam, oriunda da relaçãodecontra-alimentaçãoda teoria gerativa, em que há a subaplicação de uma certa generalização; a não-aparente-na-superfície, que é criada por processos que atuam mesmo quando não há um contexto aparente, vinculada à relação de contrassangramento, originando um efeito de superaplicação.

Dada a grande abstração do modelo derivacional, entre outros motivos, Prince e Smolensky (1993) propõem a Teoria da Otimidade (TO). Este modelo baseado em hierarquias de restrições universais, descendente direto da Gramática Harmônica (1990), é uma teoria de análise linguística híbrida, calcada no simbolismo e no conexionismo. Ao invés de tomar um input - forma subjacente - e aplicar procedimentos que o transformem em um output - forma de superfície - como a teoria até aqui descrita, na TO a ação primária, conforme McCarthy (2002), é comparativa. O verdadeiro output é membro de um conjunto de candidatos a formas de superfície. Os resultados analíticos e teóricos interessantes na TO se originam do entendimento dos detalhes de como os candidatos são comparados, comparação esta que é feita por meio da aplicação de uma hierarquia de restrições violáveis. Para a TO, a Gramática Universal (GU) constitui-se de três componentes: (i) GEN, responsável pela produção de um conjunto potencial de candidatos; (ii) EVAL, ferramenta que avalia o conjunto dado por GEN, selecionando, então, o candidato vencedor ou ótimo; e CON, que é o conjunto de restrições universais.

Atualmente, são vários os estudos que ressaltam a simplicidade e a força explanatória da hierarquia de restrições em comparação com as teorias transformacionais. As vantagens deste modelo advêm 
do seu caráter restritivo, por eliminar completamente a serialidade e o ordenamento de regras, sendo um modelo adequado para formalizar a conspiração de vários fatores na emergência de um determinado output. Contudo, segundo Costa (2007), alguns tipos de generalizações não podem ser explicados apenas pelas restrições e por seu ordenamento. Por essa razão, as generalizações que não podem ser explicadas pela teoria passam a ser o gatilho de reformulações teóricas, e nesse quadro encontram-se os casos de opacidade fonológica.

\section{Um caso de opacidade na aquisição do português brasileiro (PB), via restrições}

A opacidade está diretamente ligada a níveis intermediários de representação, ou seja, uma representação intermediária que não possa ser inferida por meio da representação fonética. Por exemplo, segundo CristófaroSilva e Gomes (2007a), uma palavra como "tristeza" pode ser produzida pela criança como [tris'teza] ou [tis'teza] em variedades do português que contemplem a palatalização. Contudo, numa palavra como "tirano", a pronúncia generalizada em dialetos palatalizantes é [tfi'ranu]. Surge, portanto, a pergunta proposta pelas autoras: como um falante de uma variedade palatalizante que seja exposto às formas "tristeza" e "tirano" sabe que a palatalização não se aplica no primeiro caso - [tis' teza] e não *[t $\int 1$ is' teza] - mas sim no segundo (Ibid., 2007, p. 10)? Em outras palavras, como o falante trata sequências sonoras idênticas nas formas de saída - ou seja [ti] em [tis'teza] e [ti'ranu] - como sequências diferentes no que concerne à palatalização $\left({ }^{*}[\mathrm{t}\right.$ fis'teza $]$, mas [tfi'ranu $\left.]\right)($ Ibid.)?

Os casos de opacidade fonológica podem ser constatados não apenas nos dados do adulto, mas também nas formas produzidas pelas crianças durante o processo de aquisição da fonologia. Segundo Matzenauer (2003), os dois casos de opacidade podem ser constatados nos dados das crianças, os quais 
poderiam perfeitamente ser explicados com base em modelos derivacionais de análise fonológica. A análise via TO torna-se, no entanto, mais complexa.

Matzenauer (2003) apresenta um caso de opacidade presente no funcionamento da classe das fricativas similar ao que trazem Cristófaro-Silva e Gomes (2007a). É indispensável salientar que, na variante do PB usada na comunidade à qual pertence o sujeito de tal estudo, a palatalização de /t/ e /d/ sempre ocorre antes de [i]. O corpus do sujeito mostra tal produção palatalizada, como podemos ver em (1).

(1)

$$
\begin{aligned}
& \text { Outputs } \\
& \text { tia ['t } \mathrm{f} \mathrm{ia}] \\
& \text { titio }\left[\mathrm{t} \int \mathrm{i} \mathrm{t} / \mathrm{iw}\right]
\end{aligned}
$$

(MATZENAUER, 2003)

A autora aponta que o caso de opacidade está presente na pronúncia de $/ \int /$ na borda esquerda da palavra fonológica, contexto no qual o sujeito apresenta, consistentemente, o emprego da plosiva coronal [t] ao invés do segmento esperado, como na pronúncia ['tika] para o item lexical xícara (Ibid., p. 95). Todavia, apesar de palatalizar a plosiva coronal em tal contexto, conforme (1), o sujeito não palataliza esse mesmo segmento antes de [i] quando é produzida em detrimento de $/ \mathrm{J} /$, como no exemplo ['tika]. Esse caso de opacidade é do tipo não-verdadeiro-na-superfície, pois o fenômeno da palatalização não está presente na produção fonética do sujeito, conforme (2).

(2)

$$
\begin{array}{lll}
\text { / } \mathrm{jikara} / & \text { /tia/ } & \\
----- & \mathrm{t} \int \mathrm{ia} & \text { palatalização da plosiva coronal diante da vogal [i] } \\
\text { tikara } & ----- & \text { plosivização de }[\mathrm{S}] \text { na borda esquerda da palavra } \\
\text { tika } & ----- & \text { apagamento de sílaba átona } \\
\text { [tika }] & {\left[\mathrm{t} \int \mathrm{ia}\right]} &
\end{array}
$$

(Ibid., p.96) 
Para os modelos transformacionais, os casos de opacidade fonológica "tornam-se transparentes ao se considerarem níveis e, portanto, ordenamentos de regras precedentes ao output..." (Ibid.). Já em modelos como a TO clássica, que adotam o mapeamento linguístico em paralelo, a formalização da opacidade pode não se mostrar de forma "transparente". Contudo, a TO pode recorrer a meios outros, oriundos da evolução do modelo, para formalizar os casos de opacidade presentes nos sistemas das línguas.

Matzenauer (2003) propõe uma análise dos dados via LPM - OT (Lexical Phonology and Morphology Optimality Theory)", de Kiparsky (2000), e via conjunção local de restrições. Salientaremos aqui a primeira abordagem, que considera a divisão entre módulo lexical e pós-lexical, remetendo à fonologia seriada.

Segundo Bonilha (2004), os diferentes níveis de representação presentes na fonologia derivacional, como na Fonologia Lexical, só existem porque se pressupõe a aplicação de regras que transformam essas representações em outras, que também serão submetidas à aplicação de mais regras. A LPM-OT, sob esse prisma, torna-se um modelo incoerente com a proposta derivacional da fonologia gerativa, motivada pela aplicação de regras, e com o berço conexionista da Teoria da Otimidade, que pressupõe o processamento em paralelo. Questiona-se, na verdade, quais seriam as vantagens de se utilizar a OT Estratal. As respostas parecem vir apenas no sentido da manutenção de modelos teóricos anteriores, como a Fonologia Lexical, que tendem a não mais serem aplicados nas análises correntes, e na tentativa de se buscar explicações para fenômenos fonológicos derivados da fonologia gerativa, como a opacidade, através da OT.

Para alguns autores, como Collischonn (2002, p. 170), qualquer corrente teórica dentro da OT não é derivacional, inclusive a LPM - OT, pois não há mais a aplicação sequencial de regras. Entendemos, no entanto, que a LPM - OT é um modelo derivacional, pois concebe 
3 IDENT-IO

(contínuo) - segmentos correspondentes

no input e no

output têm valores

idênticos para o traço

[contínuo]; IDENT-IO

(anterior) - segmentos

correspondentes no

input e no output têm

valores idênticos para

o traço [anterior];

PALATALIZAÇÃO -

uma plosiva coronal

realiza-se como

palatal antes de [i];

${ }^{*}$ ALIGN-L- (-anterior)

- não deve haver

alinhamento à esquerda de um segmento com o traço (-anterior); e

*ALIGN-L- (+contínuo)

- não deve haver

alinhamento à esquerda de um segmento com o traço (+ contínuo)

(MATZENAUER, 2003, p. 97) a aplicação, não de regras, mas de diferentes hierarquias de restrições para um determinado input - o candidato ótimo escolhido - de um ranqueamento anterior. Apesar de as restrições serem todas consideradas ao mesmo tempo na análise, o processamento em paralelo é perdido ao considerar-se a existência de níveis, representados por diferentes ordenamentos de restrições. As formas derivadas da LPM - OT nada mais são do que os outputs escolhidos que serão inputs para novos ordenamentos de restrições, como nos exemplos em (3-6), retirados de Matzenauer (2003):

Hierarquia no nível lexical ${ }^{3}$ :

PAL $>$ IDENT-IO(anterior), IDENT-IO(contínuo). > *ALIGN-L(-anterior), ${ }^{* A L I G N-L-(+c o n t i ́ n u o) ~}$

A autora aponta que essa hierarquia resulta da escolha de um output com a fricativa palatal para o input com a presença de / $/$ / (input 1 . no tableau em (4)) e a escolha de um output com a africada palatal para o input com /t/ antes de [i] (input 2. no tableau em (4)).

(4)

\begin{tabular}{|c|c|c|c|c|c|}
\hline $\begin{array}{c}\text { NÍVEL } \\
\text { LEXICAL } \\
\text { 1. / Jicara/ }\end{array}$ & $\begin{array}{c}\text { PAL } \\
* !\end{array}$ & $\begin{array}{l}\text { IDENT IO } \\
\text { (anterior) }\end{array}$ & $\begin{array}{l}\text { IDENT IO } \\
\text { (contínuo) }\end{array}$ & $\begin{array}{l}{ }^{*} \text { ALIGN-L } \\
\text { (-anterior) }\end{array}$ & $\begin{array}{l}\text { *ALIGN- L } \\
\text { (+contínuo) }\end{array}$ \\
\hline a) tica & *! & $*$ & * & & \\
\hline $\begin{array}{l}\text { b) Jica } \\
\text { c) t fica } \\
2 . / \text { tia/ }\end{array}$ & & & ${ }^{* !}$ & * & $*$ \\
\hline $\begin{array}{l}\text { a) tia } \\
\infty \text { b) } t \int \sqrt{i a}\end{array}$ & ${ }^{*} !$ & $*$ & & $*$ & \\
\hline
\end{tabular}

Na hierarquia do nível lexical, o alto ranqueamento da restrição PALATALIZAÇÃO e sua relação de dominância com as restrições de Identidade e da família ALIGN definem a escolha dos candidatos ótimos. O mesmo pode ser dito em relação à dominância de Ident sobre as restrições de alinhamento. Contudo, seguindo 
Matzenauer (Ibid.), necessitamos considerar restrições conjuntas no nível pós-lexical, as quais darão conta da emergência de uma plosiva para o segmento / $/$ / em onset silábico - quando localizado na borda esquerda da palavra - da não palatalização da plosiva que substitui a consoante /S/ e da palatalização de /t/ e /d/ quando estiverem antes de [i]. A inclusão das restrições conjuntas, no nível póslexical, é que permite formalizar o caso de opacidade que existe entre fricativas e plosivas coronais, como podemos observar na hierarquia em (5).

Nível pós-lexical:

$\left[{ }^{*}\right.$ ALIGN-L-(-anterior) \& IDENT-IO(contínuo) $] \delta>>\left[{ }^{*}\right.$ ALIGN-L-(- anterior) \& *ALIGN-L- (+contínuo)] $>>$ PAL >> IDENT-IO(anterior), IDENT-IO (contínuo) >> *ALIGN-L-(-anterior), ${ }^{*}$ ALIGN-L-(+contínuo)

Desta forma, temos o tableau em (6):

(6)

\begin{tabular}{|c|c|c|c|c|c|c|c|}
\hline $\begin{array}{l}\text { NÍVEL PÓS- } \\
\text { LEXICAL }\end{array}$ & $\begin{array}{l}\text { [ALIGN-L } \\
\text { (-ant)\& } \\
\text { IDENT IO } \\
\text { (cont)] } \delta\end{array}$ & \begin{tabular}{|c} 
ALIGN-L \\
(-ant)\& \\
ALIGN-L \\
$(-$ cont $)] \delta$
\end{tabular} & PAL & $\begin{array}{l}\text { IDENT } \\
\text { IO } \\
\text { (ant) }\end{array}$ & $\begin{array}{c}\text { IDENT } \\
\text { IO } \\
\text { (con) }\end{array}$ & $\begin{array}{c}{ }^{*} \text { ALIG } \\
\text { N-L } \\
\text { (-ant) }\end{array}$ & $\begin{array}{c}{ }^{*} \text { ALIG } \\
\text { N-L } \\
(+ \text { cont })\end{array}$ \\
\hline $\begin{array}{l}\text { 1. [ [jica] } \\
\text { a) tica } \\
\leftarrow \text { b) Sica }\end{array}$ & & ${ }^{*} !$ & ${ }^{*}$ & 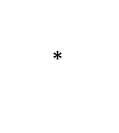 & ${ }^{*}$ & ${ }^{*}$ & * \\
\hline $\begin{array}{l}\text { c) tfica } \\
\left.\text { 2. [t } \int \mathrm{ia}\right] \\
\text { a) tia }\end{array}$ & *! & & *! & & * & * & \\
\hline ○) tfia & & & & & & * & \\
\hline
\end{tabular}

Com o último tableau, podemos observar que as formas atestadas na língua são, de fato, selecionadas. Contudo, segundo McCarthy (2007), em Costa (2007), alguns aspectos teóricos sob essa perspectiva merecem ser examinados. Em primeiro lugar, o autor presume que esse tipo de TO - serial - não seja um mecanismo suficientemente robusto para apreender a ampla gama de tipos de opacidade existente nas línguas do mundo. Por exemplo, a opacidade pode existir sob o domínio de um único estrato, e, como a opacidade é prevista por 
essa abordagem pela interação dos módulos lexical e pós-lexical, não haveria como explicar uma interação opaca que ocorresse dentro de um desses níveis. (Ibid., p. 118).

Demos um enfoque para a proposta de Kiparsky (2000), para exemplificar um dos malabarismos estruturais a que a TO foi exposta, para que o modelo pudesse dar conta de uma variedade maior de casos opacos atestados nas línguas naturais. Embora a exemplificação adotada acima seja apenas um recorte da análise de Matzenauer (2003), devemos apontar que a autora, no desenvolver completo de seu estudo, irá preferir a formalização do fenômeno opaco acima descrito somente com restrições conjuntas, em detrimento da proposta serial da TO, pois a mesma também requer o uso da conjunção local para a análise dos dados em questão.

Contudo, o uso de restrições conjuntas também é bastante criticado. (MCCARTHY, 2002, 2007; COSTA, 2007). Aparentemente, a conjunção local de restrições é capaz de apreender apenas um número muito limitado de casos de opacidade, conhecidos como contra-alimentação no foco. "Essa relação, em TO, é definida como nãoverdadeira-na-superfície. Nesse caso, um processo $X$ cria alvos potenciais para um processo $\mathrm{Y}$ aplicar, mas esse último não se aplica. É no foco, porque ambos os processos (X e Y) envolvem um mesmo segmento". (COSTA, 2007, p. 80).

Além de não ser capaz de dar conta de todos os casos de opacidade, segundo McCarthy (2002, p. 41), a conjunção de alguns tipos de restrições de fidelidade pode produzir certos tipos de pseudo-opacidade que não existem. Para o autor, a razão para esse problema decorre do ponto chave da teoria de Conjunção Local: "a opacidade real do tipo contra-alimentação é produto da interação de processos, mas a conjunção pode regular somente esses processos por proximidade. E interação e proximidade são coisas diferentes" (Ibid.). 
A proposta de Conjunção Local parece, também, enfraquecer a noção de dominação estrita defendida pela TO, pois elas refletem a união de forças de duas ou mais restrições ranqueadas abaixo na hierarquia. Atualmente, o uso das restrições conjuntas está sendo repensado com base na redefinição dos primitivos fonológicos ${ }^{4}$ e da utilização da Gramática Harmônica ${ }^{5}$ na análise dos dados.

\section{Representações linguísticas e modelo de exemplares}

Os modelos de exemplares se tornaram parte da linguística: esses são usados na fonética e fonologia (JOHNSON, 1997; PIERREHUMBERT, 2001), morfologia, sintaxe e semântica. $\mathrm{O}$ apelo deste modelo é que pode explicar fenômenos que são problemáticos em modelos derivacionais. Tais fenômenos incluem a frequência na percepção, mudança linguística gradual e as dinâmicas da aquisição da linguagem. Os modelos de exemplares têm como meta capturar a memória episódica detalhada dos eventos linguísticos que o homem retém ao armazenar exemplares com o tempo e ao comparar inputs novos àqueles já estocados.

Em um modelo de exemplares, cada categoria é representada na memória por uma nuvem larga de tokens lembrados dessa dada categoria. Essas memórias são organizadas em um mapa cognitivo, de forma que as memórias similares estão armazenadas próximas umas das outras, e memórias não semelhantes ficam distantes. $\mathrm{O}$ sistema inteiro é um mapeamento entre pontos em um espaço de parâmetro fonético e rótulos do sistema de categorização. Os rótulos, por sua vez, constituem um nível de representação autônomo ou podem ser vistos como ligações funcionais aos outros níveis de representação. É importante notarmos que os mesmos tokens lembrados podem se sujeitar a mais de um esquema de categorização sob tal modelo.
4 Veja-se Davidson (2006) para uma proposta de análise que substitui restrições conjuntas com base em traços distintivos por restrições calcadas nos primitivos da Fonologia Gestual.

5 Ver Smolensky e Legendre (2006). 
Em suma, a dinâmica de exemplares fornece um modelo incisivo dos principais achados da Fonologia de Uso (BYBEE, 2001). A suposição que o homem aprende categorias fonológicas ao lembrar vários tokens etiquetados dessas categorias explica a habilidade de aprender padrões fonéticos refinados da língua. Também explica por que padrões são incrementalmente modificados ao longo de grandes períodos de tempo na fala adulta, e por que mudanças históricas no aumento da sonoridade são tipicamente mais avançadas em palavras com grande frequência do que em palavras pouco frequentes (Ibid.).

Os exemplares foram introduzidos como um modelo psicológico por Hintzman (1986). Esses exemplares têm sido investigados no viés da percepção e da produção da fala, em que a representação de itens lexicais é vista como subjacente, formas abstratas. (JOHNSON, 1997). Cada vez que nos deparamos com uma palavra particular, estocamos a memória fonética desta palavra junto com os detalhes não linguísticos. Ao nos depararmos com uma palavra nova, esta é classificada de acordo com sua similaridade aos exemplares já estocados. Para Pierrehumbert (2001), a teoria de exemplares é uma maneira de formalizar o conhecimento do detalhe fonético que falantes têm acerca das categorias de suas línguas. A aquisição desse conhecimento pode ser interpretada em termos de uma aquisição de grandes números de traços de experiências.

\section{Opacidade na aquisição do PB, via exemplares}

Retomando os exemplos de Cristófaro-Silva e Gomes (2007a), em relação à palatalização do /t/ em uma palavra como "tirano, e da não palatalização do /t/ para o alvo lexical "tristeza" realizado como [tis'teza], ou seja, com o apagamento da líquida, observa-se que, neste caso, a "fonologia gerativa clássica sugere que, no caso de [tis'teza], a vogal [i] é opaca ao segmento [t] porque, em 
algum momento da derivação, [t] e [i] estavam separados (pela líquida [r])" (Ibid., p. 10). Embora o /r/ não seja visível na forma de superfície, este milita na representação linguística, não permitindo que palatalização ocorra.

De acordo com as autoras, na TO, as formas [tris'teza] e [ $\mathrm{t}$ is' teza] poderiam ser outputs possíveis, mas não [tis' teza]. Isso ocorre pela hierarquia de restrições da língua que origina outputs com palatalização, como os originados do ranqueamento proposto por Matzenauer (2003), evidenciado em (3). Deve-se salientar, no entanto, que, com o uso de restrições conjuntas, é possível considerar a emergência das formas [ $\left.\mathrm{t} \int \mathrm{ia}\right]$, para /tia/, e [tis'teza], para /tris'teza/, como podemos observar no tableau em (7).

(7)

\begin{tabular}{|c|c|c|c|c|c|c|}
\hline $\begin{array}{l}\text { NÍVEL PÓS- } \\
\text { LEXICAL }\end{array}$ & $\begin{array}{c}\text { [ALIGN-L } \\
\text { (-ant)\& } \\
\text { MAX } \delta\end{array}$ & PAL & $\begin{array}{c}{ }^{*} \text { Complex } \\
\text { Onset }\end{array}$ & $\begin{array}{l}\text { IDENT IO } \\
\text { (ant) }\end{array}$ & MAX & $\begin{array}{l}{ }^{*} \text { ALIG } \\
\text { N-L } \\
\text { (-ant) }\end{array}$ \\
\hline \multicolumn{7}{|l|}{ 1. /tristeza/ } \\
\hline & & & * & & & \\
\hline b) tisteza & & & & & * & \\
\hline \multirow{3}{*}{$\begin{array}{l}\text { c) t } \text { fisteza } \\
2 .\left[\mathrm{t} \int \mathrm{ia}\right] \\
\text { a) tia }\end{array}$} & ${ }^{*} !$ & & & * & & * \\
\hline & & & & & & \\
\hline & & ${ }^{*} !$ & & & & \\
\hline$\diamond \mathrm{b}) \mathrm{tfia}$ & & & & * & & * \\
\hline
\end{tabular}

Utilizando a mesma hierarquia de restrições proposta por Matzenauer (2003), com a inserção das restrições *Complex Onset e MAX I/O, a TO, novamente, formaliza um caso de opacidade na aquisição fonológica. Os custos teóricos acerca da utilização das restrições conjuntas, no entanto, permanecem os mesmos.

A palatalização é um fenômeno bastante recorrente nas línguas naturais. A figura 2 expressa a variação inerente às línguas: um conjunto de representações - neste caso fonéticas - se relacionam a uma única representação linguística categórica - neste caso uma representação fonológica. Numa abordagem fonêmica 
ou estruturalista, diríamos que o fonema /t/ se relaciona aos alofones $\left[t j, t, t \int\right]$. Tipicamente, alofonias espelham um caso de distribuição complementar em que segmentos ocorrem em contextos exclusivos (CRISTÓFARO-SILVA e GOMES, 2007a) como em (8).

(8)

\section{/ tia/ \\ ['tfia] ['tia] ['t'ia]}

Com relação à representação fonológica, os modelos multirrepresentacionais propõem que as unidades estocadas sejam as ocorrências de uso. No modelo de exemplares, todas as ocorrências percebidas são rotuladas e estocadas, criando categorias que representam diretamente a variação encontrada no uso. (CRISTÓFARO-SILVA \& GOMES, 2007b). Itens lexicais são estocados no léxico mental de acordo com as experiências do falante com a língua em questão, como já mencionado. Informações relativas ao continuum da fala também são registradas. Ou seja, ao se pronunciar uma palavra que se inicia por [pa], as palavras que apresentam esta característica são também acionadas. (PIERREHUMBERT, apud CRISTÓFARO-SILVA, 2006). Assim, o mapeamento ocorre no momento de uso, quando a linguagem é proferida e a relação entre o conteúdo sonoro e semântico se encontram. Tomamos as considerações de Cristófaro-Silva (Ibid.) acerca das representações de 'oito' e oitinho'.

A autora aponta que a palavra 'oitinho', em sua variedade dialetal, é tipicamente pronunciada como [ojtfinu] (com uma consoante africada antes doi). Contudo, pronúncias como [ojtinu] ou como [ojt $\left.\int \mathrm{i}\right]$, (dentre outras pronúncias possíveis), podem ocorrer (Ibid.). O conjunto de exemplares perceptual e de produção faz parte da nuvem de exemplares para a palavra 'oitinho'. A produção sonora da palavra 'oitinho' no Rio Grande do Sul é mais 
semelhante a uma representação como [ojtfinu] embora seja bastante improvável que nós falantes venhamos a repetir exatamente igual a palavra 'oitinho' da mesma maneira todas as vezes que a pronunciamos.

À luz de toda essa discussão, podemos relatar que o fenômeno opaco pode ser considerado como inexistente de acordo com o modo como encaramos a representação linguística. Como no exemplo em (9), ao adotarmos o modelo de exemplares, todas as possibilidades atestadas, produzidas ou apenas percebidas com e no uso da língua são estocadas e alinhavadas em nuvens de exemplares que estão próximos ou até mesmo sobrepostos. Assim, as possibilidades graduais da aquisição ou alofônicas na língua do adulto são contempladas, como em (9).
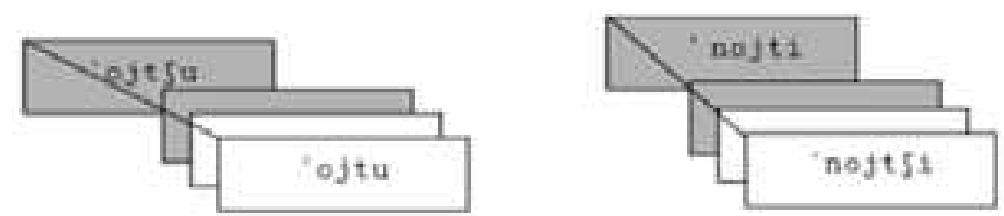

(CRISTÓFARO-SILVA \& GOMES, 2007a)

Dessa forma, a criança não produz ['tia], pois, em seu dialeto palatalizante, caso tenha tido, foram poucas as experiências linguísticas com a pronúncia do dialeto não-palatalizante, levando a criança a optar pela produção palatalizada de sua comunidade de fala. Embora as representações ricas de ['tia] e ['t $\mathrm{t}$ ia], dada sua similaridade fonética, estejam estocadas em exemplares próximos em uma mesma nuvem, a representação mais robusta é aquela cuja experiência linguística é dominante na comunidade na qual o sujeito está inserido.

Nesta mesma linha de raciocínio, podemos entender a não realização de [ $\mathrm{t} \int \mathrm{is}$ 'teza] e [' $\mathrm{t} \int \mathrm{ika}$ ], para “xícara".

Por tomarmos a representação linguística como gradual no período de aquisição, seria interessante analisarmos, com a ajuda da fonética, a oclusiva produzida em detrimento da fricativa $/ \mathrm{S} /$ em ['tika], para que 
6 Traçamos uma divisão nas vertentes da TO, pois Bonilha (2004) propôs a Teoria da Otimidade Conexionista, que, ao lidar com uma representação linguística rica, também, a princípio, não veria a opacidade como uma questão problemática. pudéssemos averiguar se esta é uma plosiva pura ou, dada a gradualidade, um segmento híbrido, contendo também características encobertas de $/ \mathrm{J} /$. Eis uma sugestão para pesquisas futuras.

Ressaltamos, assim como Cristófaro-Silva (2008b), que os resultados de pesquisas que têm como base metodológica os modelos de exemplares ainda são, muitas vezes, preliminares. Todavia, achamos importante lançar questionamentos que possam ser usados para a construção de um saber mais sólido acerca dos modelos multirrepresentacionais, como o modelo aqui defendido, e contribuir para o debate acerca da opacidade fonológica.

\section{Conclusões}

A opacidade tem um caráter abstrato e várias alternativas têm sido oferecidas para a superação deste problema. Fazemos aqui uma pequena reflexão acerca da opacidade na aquisição fonológica e de seu viés problemático para as teorias centradas no output, como a Teoria da Otimidade Gerativa ${ }^{6}$. Mostramos que os fenômenos opacos levaram a TO a um contorcionismo estrutural e a reavivar teorias que outrora já haviam sido severamente criticadas. Então, este artigo foi uma tentativa de dar suporte a uma alternativa que parece estar sendo investigada com afinco, que é um relacionamento menos distante entre a fonética e a fonologia no que concerne à própria forma de representar os fenômenos das línguas do homem. Acreditamos que o Modelo de Exemplares ao lado de modelos conexionistas, como a Teoria da Otimidade Conexionista (Bonilha, 2004) e a Gramática Harmônica, modelos que tomam a língua como inerentemente transparente e variável, são capazes de comprovar que a opacidade fonológica se encontra na complexidade do mapeamento input $\rightarrow$ outpute na pobreza da representação linguística das teorias ditas transformacionais. Embora na estrutura da TO o mapeamento em paralelo elimine a complexidade do percurso entre forma subjacente e 
forma de superfície, a representação linguística simplista, calcada na categoricidade e na homogeneidade do sistema linguístico, faz da opacidade seu ponto mais fraco. Talvez por isto, Costa (2007, p. 81) aponta que "atualmente, é consensual o fato de que a TO clássica não consegue explicar a opacidade sem mecanismos extras", em outras palavras, há a retomada da complexidade do mapeamento, entrando em um caminho serial de retomadas teóricas, para que o primado da categoricidade do sistema e, em consequência, o fenômeno da opacidade sejam mantidos. Desta forma, sugere-se que a opacidade fonológica seja uma querela dos modelos propostos para a formalização das línguas do mundo calcadas em um paradigma racionalista e no modo cartesiano como tais modelos tomam a representação linguística. 


\section{Referências}

BERMÚDEZ-OTERO, R. The acquisition of phonologial opacity. 2003. ROA.5930403 - [http://ruccs.rutgers.edu].

. Stratal Optimality Theory. (Oxford Studies in

Theoretical Linguistics) Oxford: Oxford University Press. (Ainda não publicada)

BONILHA. G. Aquisição fonológica do português brasileiro: uma abordagem conexionista da teoria da Otimidade. Tese de Doutorado: PUCRS, 2004.

BYBEE, J. The impact of us on representations: Grammar is usage and usage is Grammar. LSA Presidential Address. 2005.

. Phonology and Language Use. Cambridge Studies in Linguistics 94. Cambridge University Press, 2001.

CHOMSKY, N; HALLE, M. The Sound Pattern of English. New York: Harper Row, 1968.

COSTA, C. Opacidade e Teoria Fonológica: de regras a restrições. Tese de Doutorado: UFRGS, 2007.

COETZEE, A.; KAGER, R.; PATER, J. Introduction: phonological models and experimental data. Phonology $26-$ p. 1-8, 2009.

CRISTÓFARO-SILVA, T. Fonética e Fonologia do Português: Roteiro de estudos e guia de exercícios. São Paulo: Editora Contexto, 2008a. 
. 2008b. Desafios e Perspectivas nos Estudos da Aquisição da Linguagem. In: LETRAS. Número 36: Aquisição de Língua Materna: heterogeneidade da pesquisa. Universidade de Santa Maria. Santa Maria, 2008b, p. 113-128.

. Modelos Multi-representacionais em fonologia In: MARCHEZAN, R; CORTINA, A (Org.). Os fatos da linguagem, esse conjunto heteróclito. 1 ed. Araraquara: Cultura Acadêmica - FCL-UNESP Laboratório Editorial, 2006, v. , p. 171-186.

; GOMES, C. Representações múltiplas e organização do componente lingüístico. Fórum Lingüístico (UFSC), Florianópolis - Santa Catarina, v. 4, p. 147-177, 2007a.

; GOMES, C. Aquisição Fonológica na Perspectiva Multirepresentacional. Letras de Hoje, v. 42, p. 179-191, 2007 b.

DAVIDSON, L. Phonotactics and articulatory coordination interact in phonology: evidence from nonnative production. Cognitive Science 30:5:pp 837-862, 2006.

GOLDINGER, S. Words and voices: Episodic traces in spoken word identification and recognition memory. In: Journal of Ex-perimental Psychology: Learning, Memory \& Cognition 22(5), 1996, p. 1166-1183.

HAY, J; BRESNAN, J. 2006. Spoken syntax: The phonetics of giving a hand in New Zealand English. In: The Linguistic Review 23: 2006. 321-349

HYMAN, L. Phonology: Theory and Analysis. New York:

Holt, Rinehart \& Winston, 1975. 
HINTZMAN, D. Schema abstraction in a multiple-trace memory model. Psychological Review 93: 1986. 411-428.

ITÔ, J.; MESTER, A. Structure preservation and stratal opacity. In: LOMBARDI, L. Segmental phonology in TO. Cambridge University Press, 2001.

JOHNSON, K. Speech perception without speaker normalization: an exemplar model. In: Talker Variability in Speech Processing, K. Johnson and J. Mullenix (eds.), 145-166. Academic Press, 1997.

KAGER, R. Optimality Theory. Cambridge: Cambridge University Press, 1999.

KIPARSKY, P. Opacity and cyclicity. The Linguistic Review. 17:351-367, 2000. [online] Disponível em http://www. stanford.edu/ ${ }^{\text {kiparsky/. }}$

. Abstractness, opacity, and global rules. Bloomington, Indiana: Indiana University Linguistic Club, $1973 b$. . Paradigm effects and opacity. Ms., Stanford University, 1998.

MATZENAUER, C. Opacidade na aquisição de fricativas coronais. In: Letras de Hoje. POA: EDIPUCRS, v.38, p.233247, 2003.

. Um exemplo de opacidade na aquisição da fonologia. In: MATZENAUER, C. e BONILHA, G. Aquisição da fonologia e teoria da otimidade. Pelotas: EDUCAT, 2003.

McCARTHY, J. Sympathy and phonological opacity. Phonology. UK: Cambridge University Press, p.331-399, 1999. 
A thematic Guide to Optmality Theory. Cambridge

University Press, 2001.

. Comparative Markedness. 2002. ROA. 4890102 [http://ruccs.rutgers.edu].

Comparative Markedness. 2002. ROA. 4890102 [http://ruccs.rutgers.edu].

. Gen, Eval, and Phonological Opacity. Handout of paper present at Phonology Fest. 2006. Disponível em http://www. indiana.edu/ phono/page1/page5/page5.html.

. Hidden Generalizations: phonological opacity in Optimality Theory. Londres: Equinox, 2007.

PIERREHUMBERT, J. Exemplar dynamics: Word frequency, lenition, and contrast. In Bybee, J. and P. Hopper (eds) Frequency effects and the emergence of linguistic structure. John Benjamins: Amsterdam, 2001. 137-157.

Probalilistic Phonology: discrimination and robustness.

In: R. Bod, J. Hay, S. Jannedy (eds). 2003. 177-228.

Exemplar dynamics: Word frequency, lenition and contrast. In. J. Bybee \& P. Hopper (eds). Frequency and the emergency of linguistic structure. Amsterdam: John Benjamins, 2001. 137-157.

PRINCE, A; SMOLENSKY, P. Optimality Theory: Constraint Interaction and Generative Grammar. Report n. RuCCS-TR-2. New Brunswick, NJ: Rutgers University Center for Cognitive Science, 1993. 
SMOLENSKY, P.; LEGENDRE, G.. The harmonic mind:

From neural computation to Optimality-Theoretic grammar. Cambridge, MA: MIT Press, 2006

TIHONOVA, O. Acquisition and Opacity. Master's thesis in theoretical linguistics. University of Tromsø, 2009.

VIARIO, M; GUIMARÃES-FILHO, Z. Análise quantitativa da freqüência dos fonemas e estruturas silábicas portuguesas In: Estudos Linguísticos XXXVI (1). 2007, p. 27-36 